\title{
Polarization and Dielectric Study of Methylammonium Lead Iodide Thin Film to Reveal its Non-Ferroelectric Nature under Solar Cell Operating Conditions
}

Md Nadim Ferdous Hoque, ${ }^{1}$ Mengjin Yang, ${ }^{2}$ Zhen Li, ${ }^{2}$ Nazifah Islam, ${ }^{1}$ Xuan Pan, ${ }^{3}$ Kai Zhu, ${ }^{2 *}$ and Zhaoyang Fan $^{1 *}$

${ }^{1}$ Department of Electrical and Computer Engineering and Nano Tech Center, Texas Tech University, Lubbock, Texas, 79409, USA

${ }^{2}$ Chemistry and Nanoscience Center, National Renewable Energy Laboratory, Golden, Colorado 80401, USA

${ }^{3}$ Beijing Institute of Hydrogeology and Engineering Geology, Haidian, Beijing, 100195, China

*Contact email: kai.zhu@nrel.gov; zhaoyang.fan@ttu.edu

\begin{abstract}
Researchers have debated whether methylammonium lead iodide $\left(\mathrm{MAPbI}_{3}\right)$, with a perovskite crystal structure, is ferroelectric and therefore contributes to the current-voltage hysteresis commonly observed in hybrid perovskite solar cells (PSCs). We thoroughly investigated temperature-dependent polarization, dielectric, and impedance spectroscopies, and we found no evidence of ferroelectric effect in a $\mathrm{MAPbI}_{3}$ thin film at normal operating conditions. Therefore, the effect does not contribute to the hysteresis in PSCs, whereas the large component of ionic migration observed may play a critical role. Our temperature-based polarization and dielectric studies find that $\mathrm{MAPbI}_{3}$ exhibits different electrical behaviors below and above ca. $45{ }^{\circ} \mathrm{C}$, suggesting a phase transition around this temperature. In particular, we report the activation energies of ionic migration for the two phases and temperature-dependent permittivity of $\mathrm{MAPbI}_{3}$. This study contributes to understanding the material properties and device performance of hybrid perovskites.
\end{abstract}




\section{TOC Graphic}

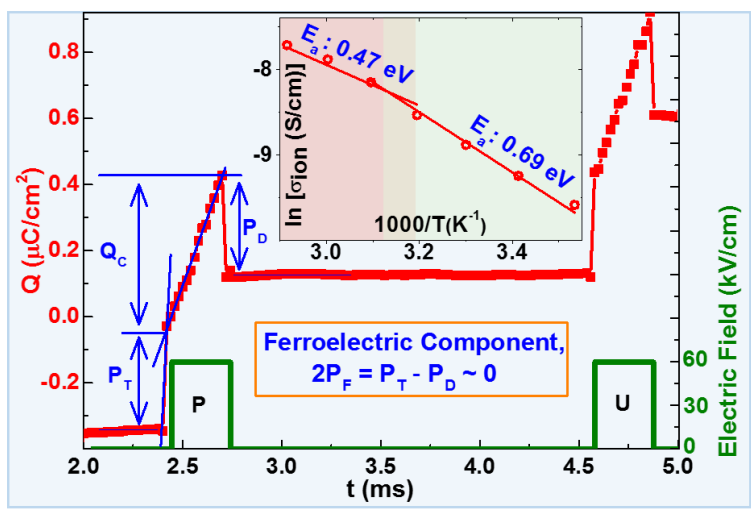


Organic-inorganic perovskite solar cells (PSCs), particularly those based on methylammonium lead iodide $\left(\mathrm{MAPbI}_{3}\right)$, have shown rapid growth, with efficiencies exceeding $20 \%{ }^{1}$ They are challenging existing silicon-based solar cell technology with their simple solution process, low fabrication cost, and possibilities of stacking with other cells for tandem structures. Along with exciting progress in terms of device efficiency, PSCs are also facing issues related to their practical application and are under careful scrutiny. One such issue is the so-called current density-voltage $(J-V)$ hysteresis phenomenon that provides a nominal efficiency depending on how the $J-V$ measurement is conducted. ${ }^{2}$ Many studies have reported an understanding of the possible nature of the hysteresis mechanism, ${ }^{3-11}$ such as the ferroelectric effect, ${ }^{12}$ ion migration, ${ }^{3}$ trapping of electronic carriers at interfaces, ${ }^{13}$ and others, as was recently reviewed. ${ }^{14}$ Each of these proposed mechanisms has strengths and weaknesses in explaining the hysteresis behavior. Further studies are necessary to understand many unresolved issues that may govern $J-V$ hysteresis and to guide the PSC design to overcome this adverse phenomenon.

Considering the commonly observed ferroelectric property of many inorganic perovskites in their tetragonal phase, it is intriguing to explore whether hybrid perovskites are ferroelectrics that contribute to $J$ - $V$ hysteresis. ${ }^{12,15,16}$ From the perspective of perovskites with tetragonal crystal structure, the polarization of $\mathrm{MAPbI}_{3}$ could be due to three components: (1) ionic polarization by the off-center shift of $\mathrm{Pb}$ in the $\mathrm{PbI}_{6}$ octahedral, ${ }^{15}$ (2) ionic polarization by the displacement of the positive charge center $\left(\mathrm{MA}^{+}\right)$from the negative charge center $\left(\mathrm{PbI}_{3}{ }^{-}\right)$, and (3) the orientation polarization of the $\mathrm{MA}^{+}$dipole. ${ }^{6}$ Conclusions from different publications, based on macroscopic polarization-electric field $(P-E)$ measurements, ${ }^{5,12,17}$ microscopic probing of ferroelectric domains, ${ }^{16,17}$ or first-principles calculations, ${ }^{6,15,18,19}$ are still being debated. At least three factors may contribute to this unsolved issue. First, $\mathrm{MA}^{+}$groups at room temperature exhibit strongly dynamic disorder ${ }^{20}$ that could invalidate the assumption in the first-principles calculations and lead to zero averaged polarization in a macro scale, even though a dipole moment might be formed at the unit-cell scale. ${ }^{6}$ Second, as a narrow-bandgap semiconductor, $\mathrm{MAPbI}_{3}$ thin films investigated typically contain a high density of charge carriers with reasonable high mobility. Such samples contribute a large conduction current during polarization measurements that may obscure the observation of a small remnant polarization - if one does exist. ${ }^{5}$ Third, the ionic conduction and its related capacitance will further complicate the analysis of the $P-E$ loop. Therefore, further 
experimental studies are still needed to determine unambiguously if $\mathrm{MAPbI}_{3}$ polycrystalline thin films have a ferroelectric property that could possibly contribute to the $J$ - $V$ hysteresis of PSCs.

A ferroelectric material shows characteristic signatures in electric-field-dependent polarization studies and temperature-dependent dielectric spectroscopy. ${ }^{21}$ For typical perovskitestructure-based ferroelectrics (e.g., perovskite oxide), in the phase transition from the hightemperature paraelectric cubic phase to the low-temperature ferroelectric tetragonal phase, the $P$ $E$ and the related $C-E$ ( $C$ : capacitance) curves also change correspondingly, giving an indication of a paraelectric to ferroelectric transition. The temperature of a $\mathrm{MAPbI}_{3}$ layer in an encapsulated PSC can easily reach more than $60{ }^{\circ} \mathrm{C}$ under typical terrestrial operating conditions, while the phase transition of $\mathrm{MAPbI}_{3}$ from a tetragonal to cubic structure occurs in this temperature range. Thus, it is also important to understand the effect of temperature on the possible ferroelectric property change and its impact on the $J-V$ hysteresis behavior. Observation of the $P-E$ loop change across the phase transition may also provide clues in determining whether a ferroelectric feature exists. Various measurements ${ }^{20,22,23}$ have shown evidence of a $\mathrm{MAPbI}_{3}$ phase change in the broad temperature range of $42{ }^{\circ} \mathrm{C} \sim 57{ }^{\circ} \mathrm{C} .{ }^{22}$ Correspondingly, the electrical and optoelectrical properties of perovskites, including the dielectric constant, ${ }^{24}$ have been reported as having a clear temperature dependence. ${ }^{25-27}$ However, the polarization response of $\mathrm{MAPbI}_{3}$ perovskite is still unknown in the typical PSC operating temperature range, and a temperature-based polarization study should provide useful information. Moreover, ferroelectric (and antiferroelectric) polar materials also show typical signatures in dielectric spectroscopy. ${ }^{21}$ Particularly, when approaching the phase transition temperature $T_{C}$ from high-temperature paraelectric to low-temperature ferroelectric, the dielectric constant will strongly increase and then decrease again below $T_{C}$. Therefore, the dielectric constant and loss tangent will exhibit a characteristic peak or jump in their temperature dependence. Thus, temperature-dependent dielectric spectroscopy provides another approach to verify the possible occurrence of ferroelectricity in hybrid perovskites.

In this work, we thoroughly investigated a temperature-dependent $P-E$ loop, and we conducted remnant polarization measurements using two different Positive(P)-Up(U)Negative(N)-Down(D), or PUND, methods. Polarization studies were further corroborated by temperature-dependent impedance and dielectric spectroscopies. All the polarization and dielectric measurements were done under dark condition to exclude the effect of any photo-induced carrier generation or recombination. Even though our macroscale studies could not exclude the possibility 
of ferroelectricity at nanoscale — and particularly at low temperature — we conclude that $\mathrm{MAPbI}_{3}$ thin films used in PSCs exhibit no apparent ferroelectric property at the normal solar cell operating temperatures $\left(10^{\circ} \mathrm{C}-70{ }^{\circ} \mathrm{C}\right)$. Therefore, ferroelectricity is not a mechanism that contributes to the $J$ - $V$ hysteresis in PSCs. Temperature-based studies reveal that $\mathrm{MAPbI}_{3}$ exhibits different electrical behaviors below and above ca. $45^{\circ} \mathrm{C}$, suggesting the phase transition around such a temperature. We found significant ionic conduction, and we report the activation energies of ionic migration for the two phases. Ionic conduction and its related capacitance contribute to the asymmetric features in the $P-E$ loop. The results unambiguously exclude ferroelectricity as one factor in governing the PSC hysteresis, whereas ionic migration contributes, if not exclusively, to the hysteresis.

The perovskite solar cell exhibits typical hysteresis in $J-V$ measurements when comparing the data taken from forward scan and reverse scan at various rates. As shown in Fig. S1 in the Supplementary Information (SI), the $J-V$ curves of a typical cell exhibit similar short-circuit current density $\left(J_{s c}\right)$ but less open-circuit voltage $\left(V_{o c}\right)$ for forward scans compared to the reverse scans, resulting in a power conversion efficiency of $15.78 \%$ (reverse scans) to $10.67 \%$ (forward scans). To determine if $\mathrm{MAPbI}_{3}$ is a ferroelectric that contributes such hysteresis in PSCs, we studied $\mathrm{MAPbI}_{3}$ thin films directly sandwiched between fluorine-doped tin oxide (FTO) and Au contacts, to avoid the potential effects of hole and electron transport layers in the polarization measurements. After cleaning the FTO glass properly, a perovskite layer was deposited by the anti-solvent method in a spin-coating process. ${ }^{28,29}$ Large crystallites of several hundred nanometers can be noticed from the top view of the scanning electron microscope (SEM) imaging of the perovskite layer (Fig. 1a). The inset is the SEM cross-sectional view of the perovskite layer sandwiched between the FTO and Au contact. The X-ray diffraction (XRD) pattern of the perovskite film (Fig. 1b) also suggests good crystallinity of the material in its tetragonal phase. The polycrystalline feature ensures that the ferroelectric polarization will be detected along the out-of-plane (vertical) orientation used in our measurements, if it does exist. For all the measurements, devices from the same batch were used to maintain conformity in various characterizations. 
To determine if $\mathrm{MAPbI}_{3}$ is a ferroelectric material, we first measured its $P-E$ hysteresis loop. Figure 1c is the schematic of our experimental setup that measures polarization charges through current integration. Unlike the conventional Sawyer-Tower configuration (Fig. S2), which may introduce error by the "back voltage" on the sensing capacitor, ${ }^{30}$ the instrument we used is based on a virtual ground configuration to minimize potential errors. As illustrated schematically in Fig. S3, when driven by a triangular waveform pulse, the measured polarization charge in time domain or its dependence on electric field exhibits characteristic profiles for ideal ferroelectrics, dielectrics, and conductors. Their field-dependent differential capacitance, $C=d Q / d V$, can also be obtained.
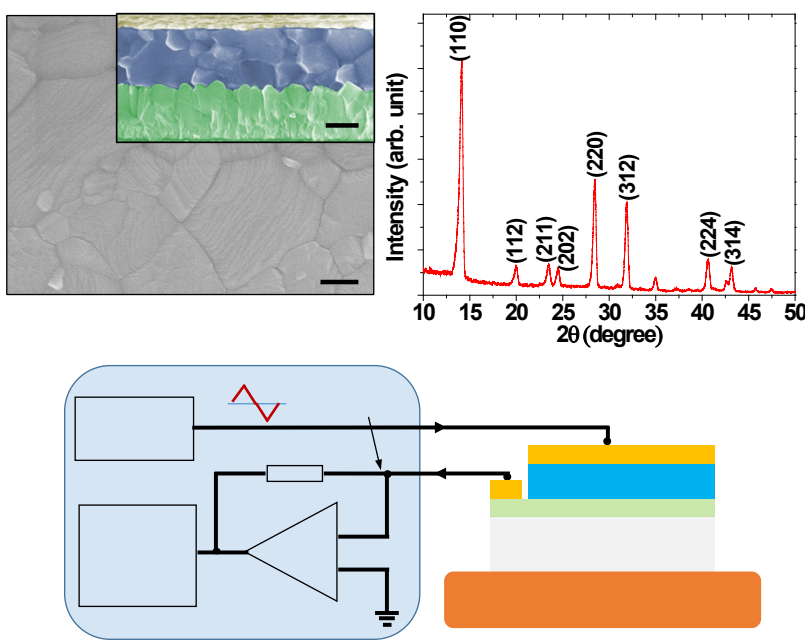

Figure 1. (a) SEM image of the surface of $\mathrm{MAPbI}_{3}$ film. The inset shows a cross-sectional view of the sample with $\mathrm{MAPbI}_{3}$ (blue) being sandwiched between FTO (green) and Au (yellow). The scale bar is $200 \mathrm{~nm}$. (b) The XRD pattern of the $\mathrm{MAPbI}_{3}$ sample on FTO, indicating the polycrystalline feature. (c) Schematic of the polarization measurement setup under controlled sample temperature.

Figure 2a is a representative polarization loop measured for the $\mathrm{MAPbI}_{3}$ perovskite sample when driven by a triangular waveform pulse with $30 \mathrm{~ms}$ pulse width and voltage amplitude of 1.2 $\mathrm{V}$ (corresponding to a field of $40 \mathrm{kV} / \mathrm{cm}$ ). Measurements at other pulse frequencies are shown in Fig. S4 with similar loop profile. Referring to Fig. S3, no obvious features of a ferroelectric material can be noticed, whereas the polarization loop seems more like a non-linear conductor-like parabolic shape. One common practice to identify a ferroelectric feature from the $P-E$ loop when a large leakage current component dominates is to study the differential capacitance, $C=d Q / d V$, 
which is also plotted in the same figure. Again, no signatures of ferroelectric capacitance can be identified from the $C-E$ plot. We also did measurements (not shown here) under a voltage of up to $3 \mathrm{~V}$, or a field up to $100 \mathrm{kV} / \mathrm{cm}$, and did not observe any features of nonlinear polarization switching. On the other hand, the parabolic-like shape indicates a dominant conducting current. The polarization curve in Fig. 2a shows asymmetric behavior, for the positive and negative voltage triangular pulse, i.e., traces 1-2 vs 3-4, and in each triangular pulse, i.e., trace 1 vs 2 , and trace 3 vs. 4, unlike the symmetric curve shown in Fig. S3b for an ideal conductor. In particular, a polarization gap opens up in the loop at $0 \mathrm{~V}$. Such a polarization loop can be approximately simulated by linear resistance and capacitance circuits, shown in Fig. 2b. In this simulation, we select $R_{1}=47 \mathrm{k} \Omega, C_{1}=0.26 \mathrm{nF}, R_{2}=145 \mathrm{k} \Omega$, and $C_{2}=0.34 \mu \mathrm{F}$, the values extracted from impedance measurement that will be discussed later. It is observed that when $C_{1}$ is connected in parallel to $R_{l}$, the symmetric mouth-shaped loop of an ideal resistor will be tilted. However, to open up a gap in the loop, a series-connected branch $R_{2}$ and $C_{2}$ has to be introduced, which is further connected in parallel with $R_{l} / / C_{l}$. As will be discussed in Fig. $4 \mathrm{c}, R_{l}$ can be modeled as the normal electron-conducting resistance in the $\mathrm{MAPbI}_{3}$ perovskite film $\left(R_{e}\right)$, and $C_{l}$ as the film bulk capacitance $\left(C_{\text {bulk }}\right)$, whereas $R_{2}$ and $C_{2}$ can be treated as ionic conduction resistance $\left(R_{i o n}\right)$ and its related capacitance $\left(C_{i o n}\right)$. From the polarization loop and the capacitance behavior, it is safe to conclude that the measured polarization response comes from a combination of conducting current along with dielectric and other capacitances. No obvious features are observed of a ferroelectric material, either remnant polarization in the $P-E$ loop, or spike in the $C-E$ plot. In addition, we noted that neither changing the frequency nor the applied electric field range results in so-called "S"shaped polarization, which was attributed as a feature of ferroelectric $\mathrm{MAPbI}_{3}{ }^{12}$ The same nonferroelectric loop was observed for the $\mathrm{Au} / \mathrm{MAPbI}_{3} / \mathrm{PEDOT}: \mathrm{PSS} / \mathrm{ITO}$ structure $^{6}$ and thus rules out the dependence of our observation on the device structure. 

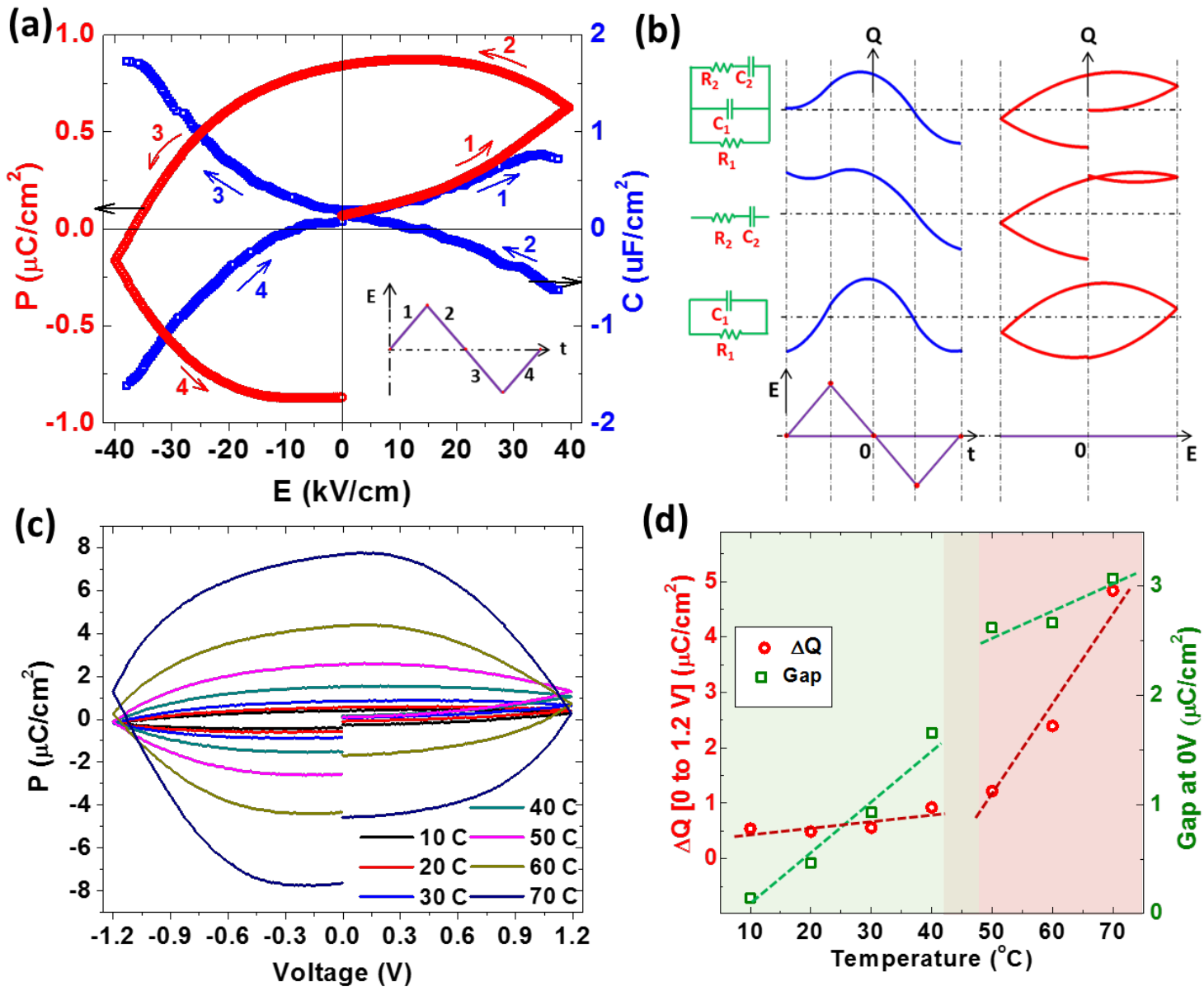

Figure 2. (a) A representative $P-E$ loop and the derived capacitance of $\mathrm{MAPbI}_{3}$ thin film when driven by a triangular voltage wave at room temperature. Arrows 1-4 indicate the swapping direction. (b) Simulation of polarization charge from different combination of $\mathrm{R}$ and $\mathrm{C}$ components in the time domain and in the format of $P$-E loop. (c) The polarization loop measured at different temperatures from $10{ }^{\circ} \mathrm{C}$ to $70{ }^{\circ} \mathrm{C}$ under same condition as in (a). (d) The measured $\Delta \mathrm{Q}$ from 0 to $1.2 \mathrm{~V}$ and the loop gap, obtained from (c), at different temperature, showing two different regions, suggesting the phase transition of $\mathrm{MAPbI}_{3}$ thin film (the dashed lines are only for visual aid).

A ferroelectric will become a paraelectric above its Curie temperature. It was reported that $P-E$ measurements conducted at low temperature down to $77 \mathrm{~K}$ showed no ferroelectric feature in $\mathrm{MAPbI}_{3}{ }^{31}{ }^{11} \mathrm{We}$ further measured the polarization in the normal working temperature range $\left(10^{\circ} \mathrm{C}-\right.$ $70{ }^{\circ} \mathrm{C}$ ) of solar cells to search for any possible ferroelectric features and also clarify the phasetransition effect. In this temperature range, $\mathrm{MAPbI}_{3}$ experiences phase transition from tetragonal to cubic crystal structure (Fig. S5), with a reported phase-transition temperature in a broad range between $42{ }^{\circ} \mathrm{C}-57{ }^{\circ} \mathrm{C}$ lacking a very sharp transition behavior. ${ }^{20,22}$ The temperature-dependent $P$ - 
$E$ loops are shown in Fig. 2c. Again, no ferroelectric behavior can be identified. As temperature increases, the measured polarization charges increase as conducting current becomes large, but their parabolic profiles have no change. This suggests that from the high-temperature cubic crystal structure to the low-temperature tetragonal structure, a transition from paraelectric to ferroelectric does not occur. Despite no sharp boundary, we observed that for temperatures below ca. $40{ }^{\circ} \mathrm{C}$, polarization charges only increase slightly with temperature, whereas above that temperature, this increment rate becomes larger. We attribute this phenomenon to the crystal phase transition of $\mathrm{MAPbI}_{3}$ perovskite that results in conductivity and capacitance change. To quantify the measured charge change with temperature, the charge generated between 0 to $1.2 \mathrm{~V}, \Delta \mathrm{Q}$, and polarization gap at different temperatures are plotted in Fig. 2(d). The graph indicates two different regions with a boundary between $40{ }^{\circ} \mathrm{C}$ and $50{ }^{\circ} \mathrm{C}$. This can be considered as the phase-transition temperature range of $\mathrm{MAPbI}_{3}$ samples measured by polarization loop.

The ferroelectric polarization from $\mathrm{MAPbI}_{3}$ could be small, if it does exist. Therefore, its contribution in the $P-E$ loop may be hidden in the dominant effect of conducting current, and may not be identified easily from the above polarization measurement. To further detect the possible ferroelectric polarization, we explored two different PUND methods. The conventional PUND method using sine pulses is shown schematically in Fig. S6a. ${ }^{32}$ In this technique, the charge contributed from the conduction current is assumed to be the same with a small value for the P and $\mathrm{U}$ pulses; therefore, during pulse $\mathrm{U}$, only the conduction charge is measured, which is deducted from the total charge measured in pulse $\mathrm{P}$ to obtain the remnant polarization $P_{r}$. The same is true for the $\mathrm{N}$ and $\mathrm{D}$ pulses. Using this method, the measured results at room temperature are shown in Fig. S6b. A zero remnant polarization charge was measured.

Conduction current could have hysteresis. So for more accurate PUND measurement without assuming equal conducting current in the two pulses, we further adopted the approach in Ref. 33, which is shown schematically in Fig. 3a, where driving pulses are in rectangular shape (constant field). For a leaky ferroelectric, the measured polarization charge consists of ferroelectric polarization $P_{F}$, dielectric polarization $P_{D}$, and charges from conduction current flow $Q_{C}$. Here, a negative poling pulse (not shown) was first applied to initialize the sample into the state of $-P_{F}$. The driving $\mathrm{P}$ pulse causes an abrupt ferroelectric polarization inversion that generates a total polarization charge of $2 P_{F}$ in the sensing circuit; it remains constant until a negative pulse (not 
shown) is applied. The $\mathrm{P}$ pulse also abruptly produces a constant dielectric polarization $P_{D}$ that disappears at the end of each pulse. Here, $P_{T}$ is defined as the sum of $2 P_{F}$ and $P_{D}$. In the duration of the $\mathrm{P}$ pulse, the field also produces a conduction current through the sample, generating a timedependent $Q_{C}$. For the very leaky $\mathrm{MAPbI}_{3}$ sample, the transient period for capacitive charging will be much shorter; on the other hand, the possible ionic screening effect may be neglected on a timescale of sub-milliseconds. ${ }^{34}$ Therefore, a constant conduction current will produce a linearly increased $Q_{C}$ with time. As shown schematically, from the measured response, which is the sum of three polarization contributions, ferroelectric polarization can be determined as $P_{F}=\left(P_{T}-P_{D}\right) / 2$ from a single pulse measurement. Our measured results, with sub-millisecond driving pulses, are shown in Fig. 3b. Again, a trivial ferroelectric polarization, with an upper limit of $5 \mathrm{nC} / \mathrm{cm}^{2}$, which is comparable with noise level, was measured for both $\mathrm{P}$ and $\mathrm{U}$ pulses. Furthermore, neither the temperature-dependent measurement nor voltage-dependent measurement, as shown in Fig. S7, suggests the existence of a ferroelectric component.
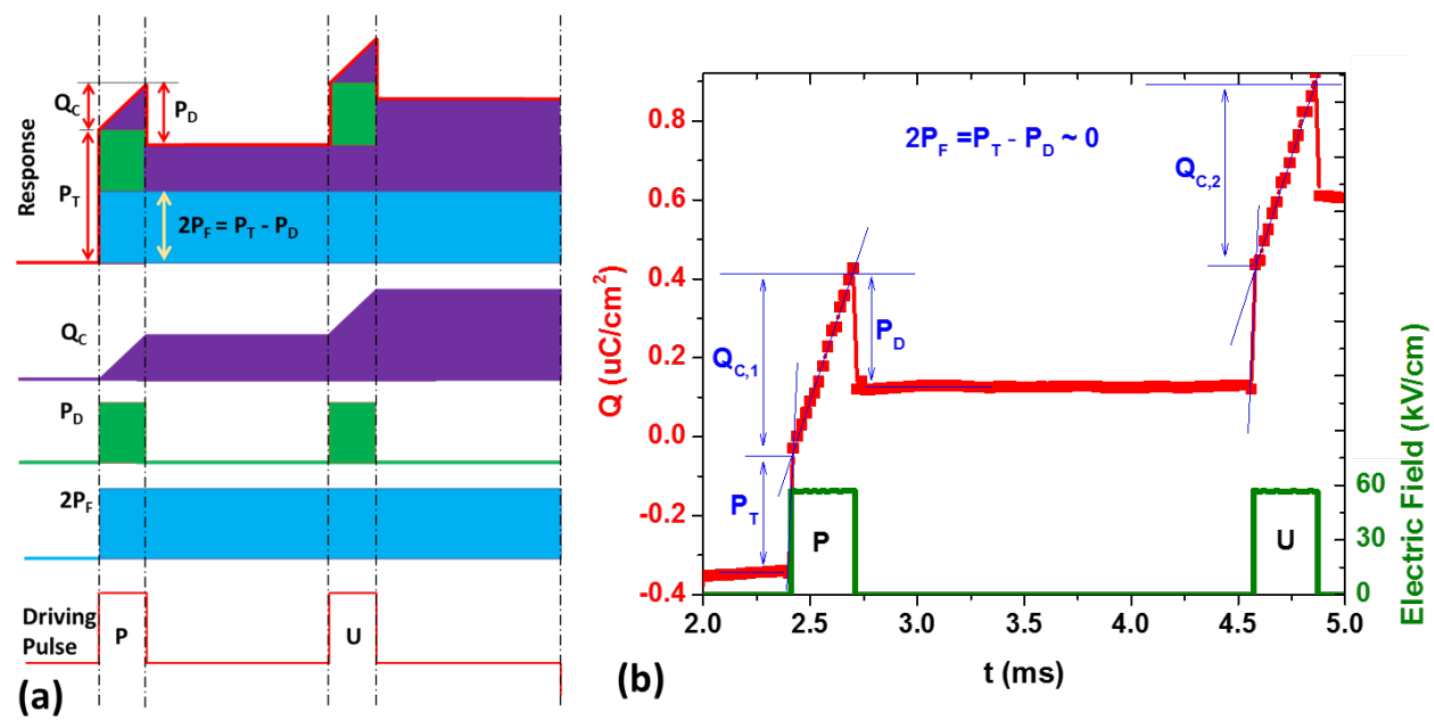

Figure 3. (a) Schematic showing the method of how to determine the ferroelectric polarization $P_{F}$. Here, after each pulse, the measured charge is not reset to zero. (b) The measured results that give a trivial $P_{F}$ value for both $\mathrm{P}$ and $\mathrm{U}$ pulses. 

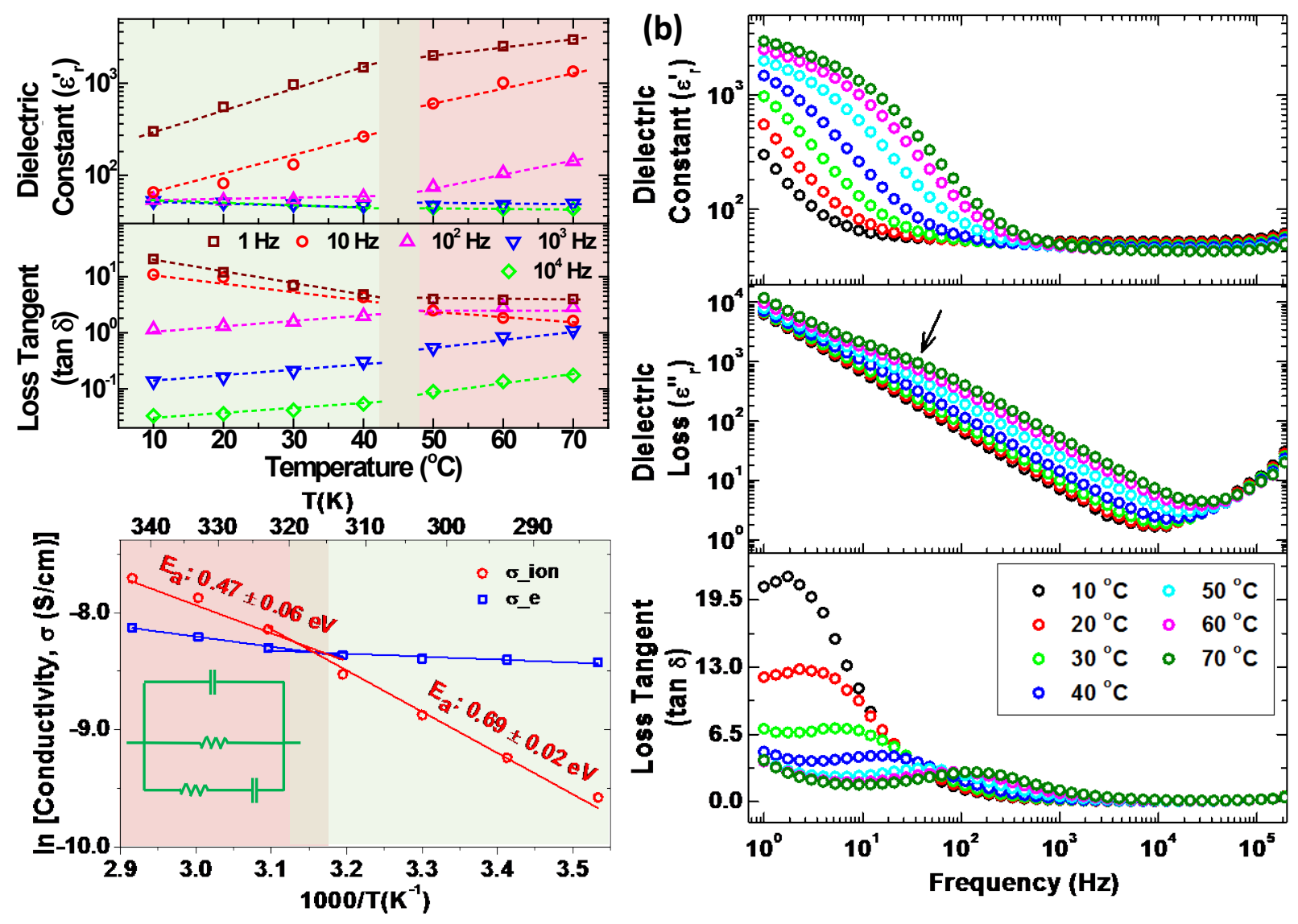

Figure 4. (a) Dependence of dielectric constant and loss tangent with temperature at specific frequencies. (b) Spectra of dielectric constant, dielectric loss, and loss tangent at different temperatures. (c) Conductivity contribution from ionic and electronic species in the perovskite at different temperatures. The inset shows the equivalent circuit to model the impedance spectrum.

Further, we measured the impedance spectra (Fig. S8) of the sample in a temperature range of $10^{\circ} \mathrm{C}-70^{\circ} \mathrm{C}$, from which we derived the frequency-dependent dielectric constant $\left(\varepsilon_{r}^{\prime}\right)$, dielectric $\operatorname{loss}\left(\varepsilon_{r}^{\prime \prime}\right)$, loss tangent $\left(\varepsilon_{r}^{\prime \prime} / \varepsilon_{r}^{\prime}\right)$, and conductivity $\left(\sigma^{\prime}\right)$. To find the possible presence of ferroelectric behavior, temperature dependence of dielectric constant and loss tangent at several selected frequencies are plotted in Fig 4a. It is known that a peak in dielectric constant and loss tangent is a characteristic of a ferroelectric material when crossing its ferroelectric-paraelectric phase transition $\left(\mathrm{T}_{\mathrm{c}}\right) .{ }^{21,35,36}$ However, no such peaks, either sharp or broad, were found in the temperature range of $10{ }^{\circ} \mathrm{C}-70{ }^{\circ} \mathrm{C}$, which covers the tetragonal-cubic phase transition temperature range. This further supports the lack of a ferroelectric feature observed in $\mathrm{MAPbI}_{3}$ thin films in the polarization studies. Moreover, two different regions, below $40{ }^{\circ} \mathrm{C}$ and above $50{ }^{\circ} \mathrm{C}$, can be observed, 
suggesting the phase change across the regions. The temperature dependence of dielectric loss and conductivity (Fig. S9) also suggests a phase transition between $40{ }^{\circ} \mathrm{C}-50{ }^{\circ} \mathrm{C}$. Figure $4 \mathrm{~b}$ shows frequency spectra of dielectric constant, dielectric loss, and loss tangent. Unusually high values of $\varepsilon_{r}^{\prime}$, similar to those in Refs. ${ }^{37,38}$, can be seen at low frequencies $\left(970\right.$ at $1 \mathrm{~Hz}$ and $30^{\circ} \mathrm{C}$ ), which dramatically decrease by more than one order at higher frequencies. Such a strong dependence of dielectric permittivity, particularly at low frequencies, is not a favorable sign of a ferroelectric nature of the material. At low frequencies, the value of $\varepsilon_{r}^{\prime}$ increases with temperature, whereas at high frequencies the trend is opposite but at a much smaller rate. This low-/high-frequency boundary is at $\sim 10^{3} \mathrm{~Hz}$ for our $\mathrm{MAPbI}_{3}$ thin film. The dielectric loss $\varepsilon_{r}^{\prime \prime}$ shows a $1 / f$ behavior for frequencies below a few $\mathrm{kHz}$, following the so-called Jonscher's law, which accurately reveals the ionic conductivity of the material. ${ }^{39,40}$ As temperature increases, a small and wide shoulder (indicated by the arrow in Fig. 4b) begins to appear and shifts toward higher frequency with temperature. Such a behavior is typical for relaxation processes, found in the generic Debye expression of permitivity. ${ }^{37}$ Considering the low frequency and broad peak feature, this relaxation process could be a Maxwell-Wagner type interfacial polarization relaxation, possibly attributed to grain boundary effects or blocking at the contacts. The dielectric loss tangent of the material was found to be high at lower frequencies, especially at lower temperature. The peak value of loss tangent shifts toward higher frequency as the temperature is increased $\left(\sim 1 \mathrm{~Hz}\right.$ for $10{ }^{\circ} \mathrm{C}$ to $\sim 200$ $\mathrm{Hz}$ for $70^{\circ} \mathrm{C}$ ). Moreover, the loss tangent starts increasing with temperature at $>100 \mathrm{~Hz}$, and below this frequency the loss tangent decreases with increasing temperature. More work is expected to be done in the future to understand this phenomenon.

As shown in Fig. S8, the Nyquist plots of impedance spectra exhibit two distinct chargetransport regimes associated with two semicircles. This is particularly obvious at higher temperatures. The first semicircle in the high-frequency regime arises from fast electron transport, whereas the second semicircle in the low-frequency regime may be attributed to slow ionic migration to the contact interface. Different models have been applied to fit the spectra to gain insight on the physical processes. Here, we employ the model previously used by Yang et al. ${ }^{38}$ and Beilsten-Edmands et al. ${ }^{5}$ in their studies of perovskite materials, to explain our measured data. It is understood that this simplified model will not capture all the subtleties but does explain the dominant processes. As shown by the equivalent circuit (inset of Fig. 4c), this model considers contributions from both electrons and mobile ions. ${ }^{38}$ Here, $R_{e}$ represents electronic resistances, and 
the ionic migration effects are modelled as a series $R_{i o n} C_{i o n}$ branch in parallel to the electronic processes. The series $C_{i o n}$ is used to model the contact blocking of mobile ions. The geometric and chemical capacitances (excluding ionic effects) are represented by $C_{b u l k}$. The fitting curves to the original measurements are shown in Fig. S10. After plotting the various ionic and electronic elements of the equivalent circuit against temperature, we observed a clear change of $R_{i o n}$ and $R_{e}$ resistivity as shown in Fig. S11. $C_{i o n}$ also showed one order of change from $10{ }^{\circ} \mathrm{C}$ to $70{ }^{\circ} \mathrm{C}$ whereas $C_{\text {bulk }}$ did not change significantly. Two different regimes for all these four parameters, although with weak contrast, can still be differentiated. This gives an indication of a soft phase transition at a temperature between $40{ }^{\circ} \mathrm{C}$ and $50{ }^{\circ} \mathrm{C}$, consistent with the measurement of polarization. Both these results confirm a similar trend of charge-conduction change between two phases. The Arrhenius plot of the extracted ionic conductivity is shown in Fig. 4c, from which the thermal activation energy for ionic conductivity in the $\mathrm{MAPbI}_{3}$ sample was derived. In the low-temperature tetragonal phase, an activation energy for ionic migration was found to be $0.69 \pm 0.02 \mathrm{eV}$, whereas it was $0.47 \pm 0.06 \mathrm{eV}$ in the high-temperature cubic phase. Our results are roughly in line with recent calculations by Eames et al., ${ }^{4}$ who reported a migration activation energy for $\mathrm{I}^{-}$vacancy of $0.58 \mathrm{eV}$ and for $\mathrm{MA}^{+}$of $0.84 \mathrm{eV}$, and by Haruyama et al., ${ }^{41}$ who reported values of 0.44 and 0.57 $\mathrm{eV}$, respectively, in a tetragonal cell. In another study, Azpiroz et al. ${ }^{11}$ calculated $0.5 \mathrm{eV}$ for $\mathrm{MA}^{+}$ ion migration. We further noticed that Yang et al. ${ }^{38}$ experimentally measured an activation energy of $0.43 \mathrm{eV}$, attributed to I' migration. But these theoretical and experimental studies did not report the activation energy differences between two phases. We speculate that the two different slopes (two different activation energies) in the Arrhenius plot were caused by the phase transition with cell volume change and atomic configuration distortion. Interestingly, it is further noticed from Fig. $4 \mathrm{c}$ that the electronic conductivity increases in the cubic phase more dramatically when compared to the tetragonal phase, suggesting a larger bandgap in the cubic phase. It was previously reported that the bandgap may be reduced from the tetragonal phase to cubic phase. ${ }^{20}$ However, our reflection spectra-based measurements, as shown in Fig. S12, indicate a positive bandgap change with temperature $\mathrm{dE}_{\mathrm{g}} / \mathrm{dT}$, in contrasting to most common semiconductors. This phenomenon was recently reported. ${ }^{26,42}$ Although there is no clear-cut phase transition from the bandgap measurement, we can still observe a larger $\mathrm{dEg} / \mathrm{dT}$ in the high-temperature regime (cubic phase). 
Ferroelectrics exhibit essential characteristic signatures in field-dependent polarization measurements and in temperature-dependent dielectric spectra across the phase transition. However, in our systematic polarization measurements and dielectric spectroscopic study, no signatures were detected; but we found significant ionic conduction. Our results suggest that $\mathrm{MAPbI}_{3}$ thin films do not exhibit ferroelectricity at a macroscale in the normal solar cell working temperature range. Other reports at low temperature ${ }^{31}$ also did not find any ferroelectric characteristic. Recent theoretical studies ${ }^{6}$ suggest that for $\mathrm{MAPbI}_{3}$, the off-center motion of $\mathrm{Pb}$ in the $\mathrm{PbI}_{6}$ octahedral is trivial, in contrast to previous calculations that included a large off-center motion component; ${ }^{15}$ therefore, only a small ferroelectric polarization could exist, contributed mainly by $\mathrm{MA}^{+}$group. However, the dynamic orientation disorder of the $\mathrm{MA}^{+}$group at normal operating temperatures may result in an average zero polarization at a macro scale, even though a dipole moment may exist in a unit cell or at nanoscale. Our different measurements confirm that no net remnant polarization exists in $\mathrm{MAPbI}_{3}$ thin film at macroscales in the PSC normal operating temperature range. Therefore, the hysteresis phenomenon in PSCs is not caused by a ferroelectric effect, but most likely is a result of ionic migration-related processes, with or without electron trapping. ${ }^{3,4,14,43}$ To gain further insight on the relationship between ionic conductivity and PSC hysteresis, temperature-dependent $J-V$ curve hysteresis of PSC was measured, as shown in Figure $\mathrm{S} 13$, indicating the hysteresis decreases when the temperature is increased from 20 to $70{ }^{\circ} \mathrm{C}$. This observation agrees with two recent studies, ${ }^{44,45}$ and is consistent with our observed faster ionic migration at higher temperature (Figure 4c), further suggesting the critical role of ionic migration in PSC hysteresis.

In summary, we studied polarization, dielectric, and impedance spectroscopies of $\mathrm{MAPbI}_{3}$ perovskite at the normal operating temperature range. We found that large conduction currents and capacitive effects govern the measured polarization charges in $\mathrm{MAPbI}_{3}$ thin films, along with a small contribution from dielectric polarization. Polarization depends on temperature and shows clear evidence of phase transition. A set of thorough macroscale polarization measurements, corroborated by temperature-dependent dielectric spectroscopic study, reveals the absence of ferroelectricity in $\mathrm{MAPbI}_{3}$ thin films under normal solar cell operating conditions. Therefore, ferroelectricity is not a factor contributing to the PSC hysteresis problem, but ionic migration, with 
or without electron trapping, should be a key factor contributing to the hysteresis. Temperaturedependent studies find that $\mathrm{MAPbI}_{3}$ exhibits different polarization, dielectric, and electrical behaviors below $40{ }^{\circ} \mathrm{C}$ and above $50{ }^{\circ} \mathrm{C}$, suggesting the phase transition from tetragonal to cubic structure across this temperature range. The activation energies of ionic migration, most likely the migration of $\mathrm{I}^{-}$vacancies, are reported for the two phases. This study will improve our understanding of $\mathrm{MAPbI}_{3}$ material properties and may also provide useful information on the ultimate solution of hysteresis-free PSCs.

\section{ACKNOWLEDGMENTS}

Funding for the work at Texas Tech University from the National Science Foundation (CBET1438681) is greatly acknowledged. The work at the National Renewable Energy Laboratory was supported by the U.S. Department of Energy under Contract No. DE-AC36-08-GO28308.

\section{Supporting Information Available:}

Experimental details, J-V curve, explanation of polarization curves, crystal structures, polarization measurements at different conditions, impedance spectra (IS), fitted parameters from IS, and absorbance spectra.

\section{References}

(1) Yang, W. S.; Noh, J. H.; Jeon, N. J.; Kim, Y. C.; Ryu, S.; Seo, J.; Seok, S. I. High-Performance Photovoltaic Perovskite Layers Fabricated through Intramolecular Exchange. Science 2015, 348, 12341237.

(2) Snaith, H. J.; Abate, A.; Ball, J. M.; Eperon, G. E.; Leijtens, T.; Noel, N. K.; Stranks, S. D.; Wang, J. T.-W.; Wojciechowski, K.; Zhang, W. Anomalous Hysteresis in Perovskite Solar Cells. J. Phys. Chem. Lett. 2014, 5, 1511-1515.

(3) Tress, W.; Marinova, N.; Moehl, T.; Zakeeruddin, S.; Nazeeruddin, M. K.; Grätzel, M. Understanding the Rate-Dependent $\mathrm{J}-\mathrm{V}$ Hysteresis, Slow Time Component, and Aging in $\mathrm{CH}_{3} \mathrm{NH}_{3} \mathrm{PbI}_{3}$ Perovskite Solar Cells: The Role of a Compensated Electric Field. Energy \& Environ. Sci. 2015, 8, 9951004. 
(4) Eames, C.; Frost, J. M.; Barnes, P. R.; O’regan, B. C.; Walsh, A.; Islam, M. S. Ionic Transport in Hybrid Lead Iodide Perovskite Solar Cells. Nat. Commun. 2015, 6, 8497.

(5) Beilsten-Edmands, J.; Eperon, G.; Johnson, R.; Snaith, H.; Radaelli, P. Non-Ferroelectric Nature of the Conductance Hysteresis in $\mathrm{CH}_{3} \mathrm{NH}_{3} \mathrm{PbI}_{3}$ Perovskite-Based Photovoltaic Devices. Appl. Phys. Lett. 2015, 106, 173502.

(6) Fan, Z.; Xiao, J.; Sun, K.; Chen, L.; Hu, Y.; Ouyang, J.; Ong, K. P.; Zeng, K.; Wang, J. Ferroelectricity of $\mathrm{CH}_{3} \mathrm{NH}_{3} \mathrm{PbI}_{3}$ Perovskite. J. Phys. Chem. Lett. 2015, 6, 1155-1161.

(7) Wu, B.; Fu, K.; Yantara, N.; Xing, G.; Sun, S.; Sum, T. C.; Mathews, N. Charge Accumulation and Hysteresis in Perovskite - Based Solar Cells: An Electro - Optical Analysis. Adv. Energy Mater. 2015, 5, 1500829.

(8) Zhao, C.; Chen, B.; Qiao, X.; Luan, L.; Lu, K.; Hu, B. Revealing Underlying Processes Involved in Light Soaking Effects and Hysteresis Phenomena in Perovskite Solar Cells. Adv. Energy Mater. 2015, 5, 1500279.

(9) van Reenen, S.; Kemerink, M.; Snaith, H. J. Modeling Anomalous Hysteresis in Perovskite Solar Cells. J. Phys. Chem. Lett. 2015, 6, 3808-3814.

(10) Zhang, Y.; Liu, M.; Eperon, G. E.; Leijtens, T. C.; McMeekin, D.; Saliba, M.; Zhang, W.; De Bastiani, M.; Petrozza, A.; Herz, L. M. Charge Selective Contacts, Mobile Ions and Anomalous Hysteresis in Organic-Inorganic Perovskite Solar Cells. Mater. Horiz. 2015, 2, 315-322.

(11) Azpiroz, J. M.; Mosconi, E.; Bisquert, J.; De Angelis, F. Defect Migration in Methylammonium Lead Iodide and Its Role in Perovskite Solar Cell Operation. Energy Environ. Sci. 2015, 8, 2118-2127. (12) Wei, J.; Zhao, Y.; Li, H.; Li, G.; Pan, J.; Xu, D.; Zhao, Q.; Yu, D. Hysteresis Analysis Based on the Ferroelectric Effect in Hybrid Perovskite Solar Cells. J. Phys. Chem. Lett. 2014, 5, 3937-3945.

(13) Shao, Y.; Xiao, Z.; Bi, C.; Yuan, Y.; Huang, J. Origin and Elimination of Photocurrent Hysteresis by Fullerene Passivation in $\mathrm{CH}_{3} \mathrm{NH}_{3} \mathrm{PbI}_{3}$ Planar Heterojunction Solar Cells. Nat. Commun. 2014, 5 , 5784.

(14) Chen, B.; Yang, M.; Priya, S.; Zhu, K. Origin of JV Hysteresis in Perovskite Solar Cells. J. Phys. Chem. Lett. 2016, 7, 905-917.

(15) Frost, J. M.; Butler, K. T.; Walsh, A. Molecular Ferroelectric Contributions to Anomalous Hysteresis in Hybrid Perovskite Solar Cells. APL Mater. 2014, 2, 081506.

(16) Kutes, Y.; Ye, L.; Zhou, Y.; Pang, S.; Huey, B. D.; Padture, N. P. Direct Observation of Ferroelectric Domains in Solution-processed $\mathrm{CH}_{3} \mathrm{NH}_{3} \mathrm{PbI}_{3}$ Perovskite Thin Films. J. Phys. Chem. Lett. 2014, 5, 3335-3339. 
(17) Xiao, Z.; Yuan, Y.; Shao, Y.; Wang, Q.; Dong, Q.; Bi, C.; Sharma, P.; Gruverman, A.; Huang, J. Giant Switchable Photovoltaic Effect in Organometal Trihalide Perovskite Devices. Nat. Mater. 2015, 14, 193-198.

(18) Stroppa, A.; Quarti, C.; De Angelis, F.; Picozzi, S. Ferroelectric Polarization of $\mathrm{CH}_{3} \mathrm{NH}_{3} \mathrm{PbI}_{3}$ : A Detailed Study Based on Density Functional Theory and Symmetry Mode Analysis. J. Phys. Chem. Lett. 2015, 6, 2223-2231.

(19) Zheng, F.; Takenaka, H.; Wang, F.; Koocher, N. Z.; Rappe, A. M. First-Principles Calculation of the Bulk Photovoltaic Effect in $\mathrm{CH}_{3} \mathrm{NH}_{3} \mathrm{PbI}_{3}$ and $\mathrm{CH}_{3} \mathrm{NH}_{3} \mathrm{PbI}_{3-\mathrm{x}} \mathrm{Cl}_{\mathrm{x}}$. J. Phys. Chem. Lett. 2014, 6, $31-$ 37.

(20) Baikie, T.; Fang, Y.; Kadro, J. M.; Schreyer, M.; Wei, F.; Mhaisalkar, S. G.; Graetzel, M.; White, T. J. Synthesis and Crystal Chemistry of the Hybrid Perovskite $\left(\mathrm{CH}_{3} \mathrm{NH}_{3}\right) \mathrm{PbI}_{3}$ for Solid-State Sensitised Solar Cell Applications. J. Mater. Chem. A 2013, 1, 5628-5641.

(21) Lines, M. E.; Glass, A. M.: Principles and Applications of Ferroelectrics and Related Materials; Oxford university press: New York, U.S.A.; 1977.

(22) Milot, R. L.; Eperon, G. E.; Snaith, H. J.; Johnston, M. B.; Herz, L. M. Temperature - Dependent Charge - Carrier Dynamics in $\mathrm{CH}_{3} \mathrm{NH}_{3} \mathrm{PbI}_{3}$ Perovskite Thin Films. Adv. Funct. Mater. 2015, 25, 62186227.

(23) Labram, J. G.; Fabini, D. H.; Perry, E. E.; Lehner, A. J.; Wang, H.; Glaudell, A. M.; Wu, G.; Evans, H.; Buck, D.; Cotta, R. Temperature-Dependent Polarization in Field-Effect Transport and Photovoltaic Measurements of Methylammonium Lead Iodide. J. Phys. Chem. Lett. 2015, 6, 35653571 .

(24) Onoda-Yamamuro, N.; Matsuo, T.; Suga, H. Dielectric Study of $\mathrm{CH}_{3} \mathrm{NH}_{3} \mathrm{PbX}_{3}(\mathrm{X}=\mathrm{Cl}, \mathrm{Br}, \mathrm{I}) . J$. Phys. Chem. Solids 1992, 53, 935-939.

(25) Jiang, Y.; Soufiani, A. M.; Gentle, A.; Huang, F.; Ho-Baillie, A.; Green, M. A. Temperature Dependent Optical Properties of $\mathrm{CH}_{3} \mathrm{NH}_{3} \mathrm{PbI}_{3}$ Perovskite by Spectroscopic Ellipsometry. Appl. Phys. Lett. 2016, 108, 061905.

(26) Foley, B. J.; Marlowe, D. L.; Sun, K.; Saidi, W. A.; Scudiero, L.; Gupta, M. C.; Choi, J. J. Temperature Dependent Energy Levels of Methylammonium Lead Iodide Perovskite. Appl. Phys. Lett. 2015, 106, 243904.

(27) Yamada, Y.; Nakamura, T.; Endo, M.; Wakamiya, A.; Kanemitsu, Y. Near-Band-Edge Optical Responses of Solution-Processed Organic-Inorganic Hybrid Perovskite $\mathrm{CH}_{3} \mathrm{NH}_{3} \mathrm{PbI}_{3}$ on Mesoporous $\mathrm{TiO}_{2}$ Electrodes. Appl. Phys. Express 2014, 7, 032302. 
(28) Xiao, M.; Huang, F.; Huang, W.; Dkhissi, Y.; Zhu, Y.; Etheridge, J.; Gray - Weale, A.; Bach, U.; Cheng, Y. B.; Spiccia, L. A Fast Deposition - Crystallization Procedure for Highly Efficient Lead Iodide Perovskite Thin - Film Solar Cells. Angew. Chem. 2014, 126, 10056-10061.

(29) Islam, N.; Yang, M.; Zhu, K.; Fan, Z. Mesoporous Scaffolds Based on $\mathrm{TiO}_{2}$ Nanorods and Nanoparticles for Efficient Hybrid Perovskite Solar Cells. J. Mater. Chem. A 2015, 3, 24315-24321.

(30) Almora, O.; Guerrero, A.; Garcia-Belmonte, G. Ionic Charging by Local Imbalance at Interfaces in Hybrid Lead Halide Perovskites. Applied Physics Letters 2016, 108, 043903.

(31) Zhao, Y.; Liang, C.; Zhang, H.; Li, D.; Tian, D.; Li, G.; Jing, X.; Zhang, W.; Xiao, W.; Liu, Q. Anomalously Large Interface Charge in Polarity-Switchable Photovoltaic Devices: An Indication of Mobile Ions in Organic-Inorganic Halide Perovskites. Energy Environ. Sci. 2015, 8, 1256-1260.

(32) Fukunaga, M.; Noda, Y. New Technique for Measuring Ferroelectric and Antiferroelectric Hysteresis Loops. J. Phys. Soc. Jpn. 2008, 77, 064706.

(33) Naganuma, H.; Inoue, Y.; Okamura, S. Evaluation of Electrical Properties of Leaky $\mathrm{BiFeO}_{3}$ Films in High Electric Field Region by High-Speed Positive-Up-Negative-Down Measurement. Appl. Phys. Express 2008, 1, 061601.

(34) Yuan, Y.; Chae, J.; Shao, Y.; Wang, Q.; Xiao, Z.; Centrone, A.; Huang, J. Photovoltaic Switching Mechanism in Lateral Structure Hybrid Perovskite Solar Cells. Adv. Energy Mater. 2015, 5, 1500615.

(35) Qiao, L.; Bi, X.: Epitaxial Integration of Ferroelectric $\mathrm{BaTiO}_{3}$ with Semiconductor Si: From a Structure-Property Correlation Point of View. In Ferroelectrics - Material Aspects; Lallart, M., Ed.; INTECH Open Access Publisher, 2011.

(36) Lunkenheimer, P.; Krohns, S.; Gemander, F.; Schmahl, W.; Loidl, A. Dielectric Characterization of a Nonlinear Optical Material. Sci. Rep. 2014, 4, 6020.

(37) Juarez-Perez, E. J.; Sanchez, R. S.; Badia, L.; Garcia-Belmonte, G.; Kang, Y. S.; Mora-Sero, I.; Bisquert, J. Photoinduced Giant Dielectric Constant in Lead Halide Perovskite Solar Cells. J. Phys. Chem. Lett. 2014, 5, 2390-2394.

(38) Yang, T. Y.; Gregori, G.; Pellet, N.; Grätzel, M.; Maier, J. The Significance of Ion Conduction in a Hybrid Organic - Inorganic Lead - Iodide - Based Perovskite Photosensitizer. Angew. Chem. 2015, 127, 8016-8021.

(39) Jonscher, A. Analysis of the Alternating Current Properties of Ionic Conductors. J. Mater. Sci. 1978, 13, 553-562.

(40) Lin, Q.; Armin, A.; Nagiri, R. C. R.; Burn, P. L.; Meredith, P. Electro-Optics of Perovskite Solar Cells. Nat. Photonics 2015, 9, 106-112. 
(41) Haruyama, J.; Sodeyama, K.; Han, L.; Tateyama, Y. First-Principles Study of Ion Diffusion in Perovskite Solar Cell Sensitizers. J. Am. Chem. Soc. 2015, 137, 10048-10051.

(42) Leong, W. L.; Ooi, Z. E.; Sabba, D.; Yi, C.; Zakeeruddin, S. M.; Graetzel, M.; Gordon, J. M.; Katz, E. A.; Mathews, N. Identifying Fundamental Limitations in Halide Perovskite Solar Cells. Adv. Mater. 2016, 28, 2439-2445.

(43) Meloni, S.; Moehl, T.; Tress, W.; Franckevičius, M.; Saliba, M.; Lee, Y. H.; Gao, P.;

Nazeeruddin, M. K.; Zakeeruddin, S. M.; Rothlisberger, U. Ionic Polarization-Induced Current-Voltage Hysteresis in $\mathrm{CH}_{3} \mathrm{NH}_{3} \mathrm{PbX}_{3}$ Perovskite Solar Cells. Nat. Commun. 2016, 7, 10334.

(44) Ono, L. K.; Raga, S. R.; Wang, S.; Kato, Y.; Qi, Y. Temperature-Dependent Hysteresis Effects in Perovskite-Based Solar Cells. J. Mater. Chem. A 2015, 3, 9074-9080.

(45) Yu, H.; Lu, H.; Xie, F.; Zhou, S.; Zhao, N. Native Defect-Induced Hysteresis Behavior in Organolead Iodide Perovskite Solar Cells. Adv. Funct. Mater. 2016, 26, 1411-1419. 